\title{
Exercise-Induced Vasculitis in a Patient With Negative Ultrasound Venous Reflux Study: A Mimic of Stasis Dermatitis
}

\author{
Swaminathan Sundaresan, MD; Michael R. Migden, MD; Sirunya Silapunt, MD
}

\section{PRACTICE POINTS}

- Clinical history of a transient nature is the mainstay in the diagnosis of exercise-induced vasculitis.

- Exercise-induced vasculitis largely is documented in photographs or by history and may be misdiagnosed as stasis dermatitis due to its clinical morphology and lower leg location.

- Dermatologists should be aware of this disorder and consider performing further workup to rule out stasis dermatitis and diagnose this mimic.

- Preventative measures are superior to treatment and mainly include compression therapy.

To the Editor:

The transient and generic appearance of exercise-induced vasculitis (EIV) makes it a commonly misdiagnosed condition. The lesion often is only encountered through photographs brought by the patient or by taking a thorough history. The lack of findings on clinical inspection and the generic appearance of EIV may lead to misdiagnosis as stasis dermatitis due to its presentation as erythematous lesions on the medial lower legs.

A 68-year-old woman with no notable medical history was referred to our clinic for suspected stasis dermatitis. At presentation, no lesions were identified on the legs, but she brought photographs of an erythematous urticarial eruption on the medial lower legs, extending from just above the sock line to the mid-calves (Figure). The eruptions had occurred over the last 16 years, typically presenting suddenly after playing tennis or an extended period of walking and spontaneously resolving in 4 days. The lesions were painless, restricted to the calves, and were not pruritic, though the initial presentation 16 years prior included pruritic pigmented patches on the anterior thighs. Because the condition spontaneously improved within days, no treatment was attempted. An ultrasound venous reflux study ruled out venous reflux and stasis dermatitis.

Our patient stated that her 64-year-old sister had reported the same presentation over the last 8 years. Her physical activity was limited strictly to walking, and the lesions occurred after walking for many hours during the day in the heat, involving the medial aspects of the lower legs extending from the ankles to the full length of the calves. Her eruption was warm but was not painful or pruritic. It resolved spontaneously after 5 days with no therapy.

Our patient was advised to wear compression stockings as a preventative measure, but she did not adhere to these recommendations, stating it was impractical to wear compression garments while playing tennis.

Exercise-induced vasculitis most commonly is seen in the medial aspects of the lower extremities as an erythematous urticarial eruption or pigmented purpuric plaque rapidly occurring after a period of exercise. ${ }^{1,2}$ Lesions often are symmetric and can be pruritic and painful with a lack of systemic symptoms. ${ }^{3}$ These generic clinical manifestations may lead to a misdiagnosis of stasis dermatitis.

Drs. Sundaresan and Silapunt are from the University of Texas McGovern Medical School, Houston. Dr. Silapunt is from the Department of Dermatology. Dr. Migden is from the Departments of Dermatology and Head and Neck Surgery, University of Texas MD Anderson Cancer Center, Houston. The authors report no conflict of interest.

Correspondence: Sirunya Silapunt, MD, 6655 Travis St, Ste 980, Houston, TX 77030 (Sirunya.Silapunt@uth.tmc.edu).

doi:10.12788/cutis.0186 

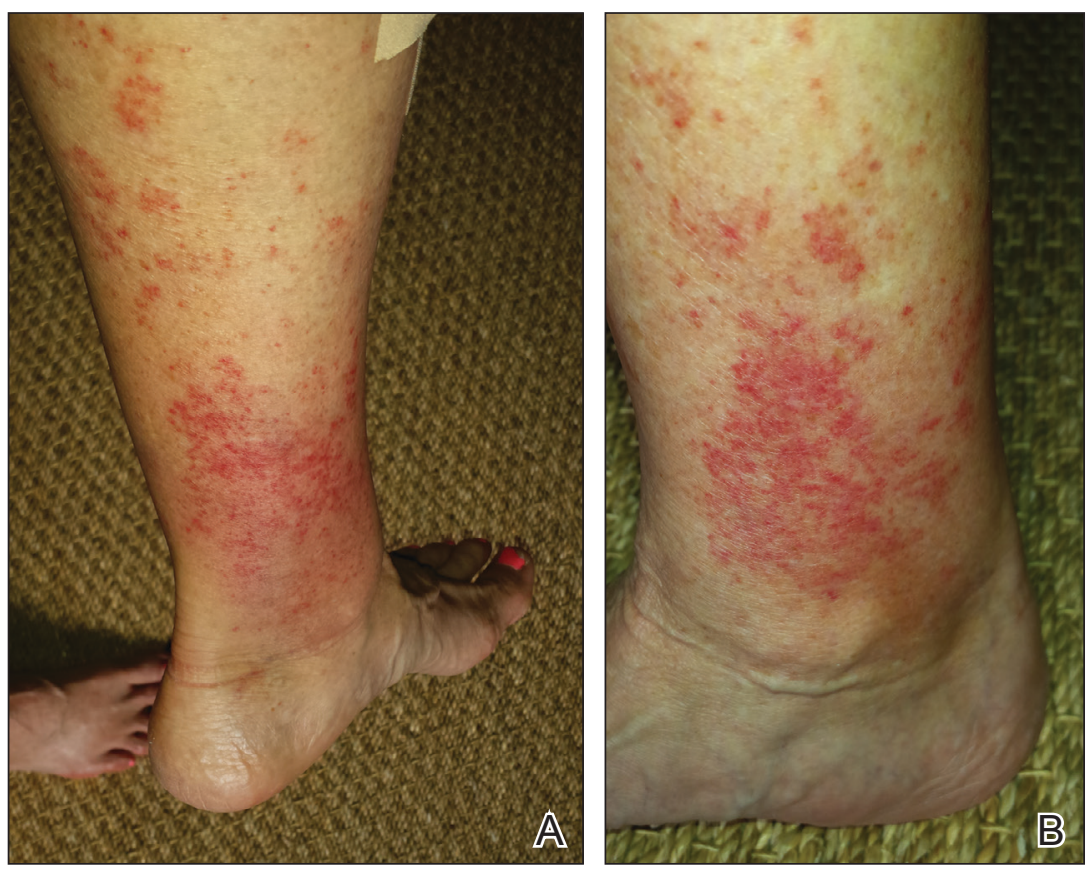

Exercise-induced vasculitis. A, Erythematous purpuric lesions on the medial aspect of the left lower leg with a distal linear delineation at the sock line. B, Urticarial erythematous eruption on the medial aspect of the right lower leg.
One case report included initial treatment of presumptive cellulitis. ${ }^{4}$ Important clinical findings include a sparing of skin compressed by tight clothing such as socks, a lack of systemic symptoms, rapid appearance after exercise, and spontaneous resolution within a few days. No correlation with chronic venous disease has been demonstrated, as EIV can occur in patients with or without chronic venous insufficiency. ${ }^{5}$ Duplex ultrasound evaluation showed no venous reflux in our patient.

The pathophysiology of EIV remains unknown, but the concept of exercise-altered microcirculation has been proposed. Heat generated from exercise is normally dissipated by thermoregulatory mechanisms such as cutaneous vasodilation and sweat. ${ }^{1,6}$ When exercise is extended, done concomitantly in the heat, or performed in legs with preexisting edema or substantial adipose tissue that limit heat attenuation, the thermoregulatory capacity is overloaded and heat-induced muscle fiber breakdown occurs. ${ }^{1,7}$ Atrophy impairs the skeletal muscle's ability to pump the increased venous return demanded by exercise to the heart, leading to backflow of venous return and eventual venous stasis. ${ }^{1}$ Reduction of venous return together with cutaneous vasodilation is thought to induce erythrocyte extravasation.

Histologic examination demonstrates features of leukocytoclastic vasculitis with perivascular lymphocytic and neutrophilic infiltrates. ${ }^{2}$ Erythrocyte extravasation, IgM deposits, and identification of C3 also have been reported. ${ }^{8,9}$ The spontaneous resolution of EIV has led to treatment efforts being focused on preventative measures. Several cases have reported some degree of success in preventing EIV with compression therapy, venoactive drugs, systemic steroids, and application of topical steroids before exercise. ${ }^{3}$

The clinical morphology and lower leg location of EIV leads to a common misdiagnosis of stasis dermatitis. Clinical history of a transient nature is the mainstay in the diagnosis of EIV, and ultrasound venous reflux study may be required in some cases. Preventative measures are superior to treatment and mainly include compression therapy.

\section{REFERENCES}

1. Ramelet AA. Exercise-induced vasculitis. J Eur Acad Dermatol Venereol. 2006;20:423-427.

2. Kelly RI, Opie J, Nixon R. Golfer's vasculitis. Australas J Dermatol. 2005;46:11-14.

3. Ramelet AA. Exercise-induced purpura. Dermatology. 2004;208:293-296.

4. Cushman D, Rydberg L. A general rehabilitation inpatient with exercise-induced vasculitis. PM R. 2013;5:900-902.

5. Veraart JC, Prins M, Hulsmans RF, et al. Influence of endurance exercise on the venous refilling time of the leg. Phlebology. 1994;23:120-123.

6. Noakes T. Fluid replacement during marathon running. Clin J Sport Med. 2003;13:309-318.

7. Armstrong RB. Muscle damage and endurance events. Sports Med. 1986;3:370-381.

8. Prins M, Veraart JC, Vermeulen AH, et al. Leucocytoclastic vasculitis induced by prolonged exercise. Br J Dermatol. 1996;134:915-918.

9. Sagdeo A, Gormley RH, Wanat KA, et al. Purpuric eruption on the feet of a healthy young woman. "flip-flop vasculitis" (exercise-induced vasculitis). JAMA Dermatol. 2013;149:751-756. 